\title{
Applying the Multisim Technology to Teach the Course of High Frequency Power Amplifier
}

\author{
Gang Lv \& Yuan-Sheng Xue \\ School of Electronic Information, Soochow University, Suzhou 215006, China \\ Tel: 86-512-6219-0898Ｅ-mail: lvgang@suda.edu.cn \\ ${ }^{2}$ Suzhou Software Industry Developing Office, No.979 Suzhou RenMin Road, Suzhou 215002, China \\ Tel: 86-512-6676-7393 E-mail: xys06@126.com
}

The research is supported by the project of the Higher Education Teaching Reform Engineering of Soochow University (No. 31315733). (Sponsoring information)

\begin{abstract}
As one important professional base course in the electric information specialty, the course of "high frequency electronic circuit" has strong theoretical characteristic and abstract content. To enhance the teaching quality of this course, the computer simulation technology based on Multisim is introduced into the teaching of "high frequency electronic circuit", which could not only deepen students' understanding and memory of basic concepts, but also effectively help students to understand and grasp the principle of the high frequency circuit by the circuit design module and the simulation analysis function. The teaching application effect of the high frequency class $\mathrm{C}$ power amplifier has also proved that the Multisim software could realize the organic combination of theory and practice, and it is the profitable supplement of the traditional teaching mode.
\end{abstract}

Keywords: Multisim, High frequency power amplifier, Simulation teaching

\section{Introduction}

High frequency electronic circuit is the important basic course of many specialties such as electronics, information, and communication (Zhang, 1993). This course has strong theoretical character, abstract content, complex computation, and high requirement about students' practice ability. Traditional teaching emphasizes the mathematical derivation, and students' attention is centralized in the memory of large numerous of theoretical formula, so most of them feel difficult to study this course. At the same time, many of them could not really understand the application of these theories in the practice, so they will obtain high scores and low abilities. To stimulate students' learning interest and enhance the teaching quality and practice ability of the course, it is imperative to introduce the EDA technology into the teaching of the "High Frequency Electronic Circuit".

As a special EDA tool software used in the simulation and design of electronic circuit (Nie, 2007), Multisim has many advanced functions such as the simulation and design of high frequency, which are not contained in current numerous EDA software. On the one hand, the ideal model library provided by this software could quickly establish the simple circuit, and the clear simulation figure could deepen students' understanding and memory to basic concepts. On the other hand, the practice circuit model provided by the Multisim technology could help students to know the high frequency experiment circuit well, and know which parameters should be adjusted and which faults will occur when testing the actual circuit, so students could have definite object in view to make the circuit experiment (Lao, 2007). Combining with the high frequency class $\mathrm{C}$ power amplifier, the application of the Multisim software will be introduced in the teaching of high frequency electronic circuit as follows.

\section{High frequency class $\mathbf{C}$ power amplifier}

High frequency class C power amplifier is the basic unit circuit in the high frequency electronic circuit (Liu, 2004), and it has been widely used in many electronic equipments such as broadcast, radar, and communication. The working principle and characteristics of the class $\mathrm{C}$ power amplifier are the emphases and difficulties in the teaching of the high frequency electronic circuit.

Generally, the class C power amplifier includes three working states, i.e. under-voltage, critical voltage, and overvoltage. These three working states are related with the change of the extent of the input signal Ubm, the power voltage Vcc, and the load R. Their dynamic characteristic curve is seen in Figure 1.

In traditional teaching course, teachers could not demonstrate above abstract and statistic changing curves dynamically to students, so students could only rote these changing curves in the learning process, and because lacking in the changing course of the dynamic characteristic curve, the memory is very difficult. 


\section{Applying the Multisim technology to demonstrate and validate the characteristics of the class $C$ power amplifier}

The class $\mathrm{C}$ amplifier circuit is seen in Figure 2 (Zhou, 2006). The circuit is composed by the front-level small signal amplified circuit and the back-level class $\mathrm{C}$ amplified circuit. The front-level circuit adopts the base-bias bleeder circuit, and the back-level circuit adopts the self-bias circuit. The loading circuit selects the $\pi$-type network, which could not only realize the frequency selection filtering, but also realize the function of impedance matching. In the front-level, R1 and R2 provide the base bias for the dynatron Q1, R3 and C1 provide a DC negative feedback to stabilize its static working point, and the initial loops of $\mathrm{T} 1$ and $\mathrm{C} 2$ compose the parallel resonance circuit. Through transforming and coupling, the amplified signals will change the drive signals of the dynatron Q2 on L1, and the dynatron Q2 adopts the self-bias circuit, and C6, C7, and L4 compose a $\pi$-type matching network, and R4 is the antenna load.

$\mathrm{Vcc}$ is the DC voltage source of $12 \mathrm{~V}$, and $\mathrm{V} 1$ is the AC signal source with the extent of $100 \mathrm{mV}$ (effective value) and the frequency of $10 \mathrm{MHz}$. In the simulation, the Q2 back-level loop is interrupted first, and by adjusting C2, the front-level small signal amplified circuit will be resonated on the basic frequency of $10 \mathrm{MHz}$, and then the back-level class $\mathrm{C}$ amplified circuit will be connected to run the simulation.

\subsection{Modulating characteristic of collector}

The collector modulating characteristic means the relationship between the class $\mathrm{C}$ collector current with Vcc when the parameters $\mathrm{Ubm}$ and $\mathrm{R}$ are not changeable.

The transient analysis function provided in Multisim could observe the changing rules of the collector current when the collector voltages respectively are 12V, 26V, and 30V. Singly click "Simulate-Analysis- Transient-Analysis", and open the dialog frame of "Transient Analysis", and set up the beginning analysis time for $2.035 \mathrm{e}-005 \mathrm{~s}$, and the ending analysis time for $2.085 \mathrm{e}-005 \mathrm{~s}$, and in the Output, the analyzed object should be selected, and by singly clicking the "Simulate", the simulation result of analyzing could be obtained as seen in Figure 3.

When Vcc increases, the amplifier enters into the under-voltage region from the over-voltage region, and the waveform of the current changes from the concave crown cosine impulse to the cosine impulse, and in the under-voltage region, the extent of the current is almost constant. In above Figure, when Vcc $=12 \mathrm{~V}$, the circuit is in the under-voltage state, and when $\mathrm{Vcc}=26 \mathrm{~V}$, the circuit is in the critical state.

\subsection{Amplitude characteristic}

The amplitude characteristic means the relationship between the current of the class $\mathrm{C}$ collector with the input signal Ubm when the parameters Vcc and $\mathrm{R}$ are constant.

The function of transient analysis could be used to observe the change rules of the collector current when the circuit input energizing quantities respectively are $40 \mathrm{mV}, 70 \mathrm{mV}$, and $100 \mathrm{mV}$. Singly click "Simulate-AnalysisTransient-Analysis", and open the dialog frame of "Transient Analysis", and set up same parameters with the above, and by singly clicking the "Simulate", the simulation result of analyzing could be obtained as seen in Figure 4.

The result indicates that with the increase of Ubm, the amplifier will enter into the over-voltage region from the under-voltage region. And when Ubm increases to the over-voltage region, though the cosine pulse amplitude increase, the concave crown will also increase.

\subsection{Loading characteristic}

The loading characteristic means the relationship between the collector current and the loading $\mathrm{R}$ of the high frequency power amplifier when the parameters such as Ubm and Vcc are constant.

The function of transient analysis could be used to observe the changing rules of the collector current when the circuit loads respectively are $10 \Omega, 50 \Omega$, and $100 \Omega$. Singly click "Simulate-Analysis- Transient-Analysis", and open the dialog frame of "Transient Analysis", and set up same parameters with the above, and by singly clicking the "Simulate", the simulation result of analyzing could be obtained as seen in Figure 5.

The result indicates that when $\mathrm{R}$ increases gradually, the amplifier enters into the over-voltage region from the under-voltage region. In the under-voltage region, the maximum value Icm of the collector current pulse changes little, but descend a little. And in the over-voltage region, the current of collector begins to become concave, and the concave degree will drastically increase with the increase of $\mathrm{R}$, so the Icm will drastically descend.

The experiment simulation of above three parameters could help students more profoundly understand the working characteristic of the class $\mathrm{C}$ amplifier by the virtual mode, enhance their understanding about the class $\mathrm{C}$ circuit, increase their understanding and memories to the basic concepts, and eventually enhance the teaching quality. 


\section{Conclusions}

From the above class $\mathrm{C}$ circuit simulation example, on the one hand, the Multisim software could provide ideal model with ideal simulation waveform, and deepen students' understanding and memory for theoretical knowledge. On the other hand, it could provide the design model of practical circuit, which could help students to quickly and flexibly modify and adjust the circuit parameters, and observe the change of waveform and research the causes, and cultivate students' practice interests, and enhance their innovational ability. Therefore, the application of the Multisim software in the course of high frequency electronic circuit could improve the organic combination of theory and practice, which is the profitable supplement of traditional teaching method.

Since 2007, the university has reformed the course of high frequency electronic circuit, and introduced the Multisim software into the classroom to assist the teaching. By the organic combination between the virtual simulation experiment and the theoretical teaching, students generally reflect they could understand the basic concepts and methods in this course better, and the pass rate of this course has been enhanced from $71 \%$ to $83 \%$, with good teaching effect.

\section{References}

Lao, Wuyi \& Lao, Jia. (2007). Analysis/ Design and Simulation of the Simulated Electronic Circuit. Beijing: Tingshua University Press. May of 2007.

Liu, Zhengyu. (2004). Design and Making of Electronic Circuit. Fuzhou: Fujian Science \& Technology Publishing House. April of 2004.

Nie, Dian. (2007). Application of the Multisim9 Computer Simulation in the Electric Circuit Design. Beijing: Electronic Industry Press. June of 2007.

Zhang, Suwen \& Lu, Zhaoxiong. (1993). High-frequency Electronic Circuit. Beijing: Higher Education Press. April of 1993.

Zhou, Minglai, Xia, Chun \& Lv, Gang. (2006). Guidance of Electronic Circuit Experiment. Suzhou: Suzhou University Press. August of 2006.
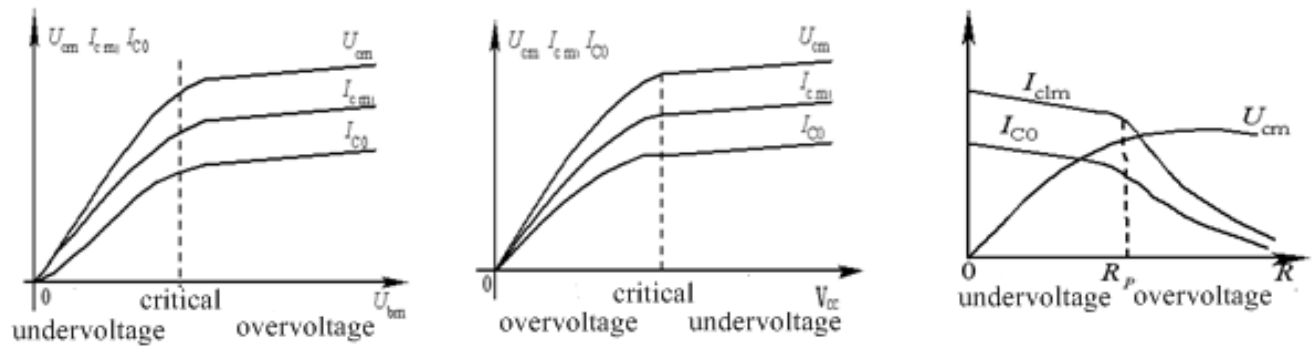

Figure 1. Icm and Ucm Changing Curves When Ubm, Vcc, and R change

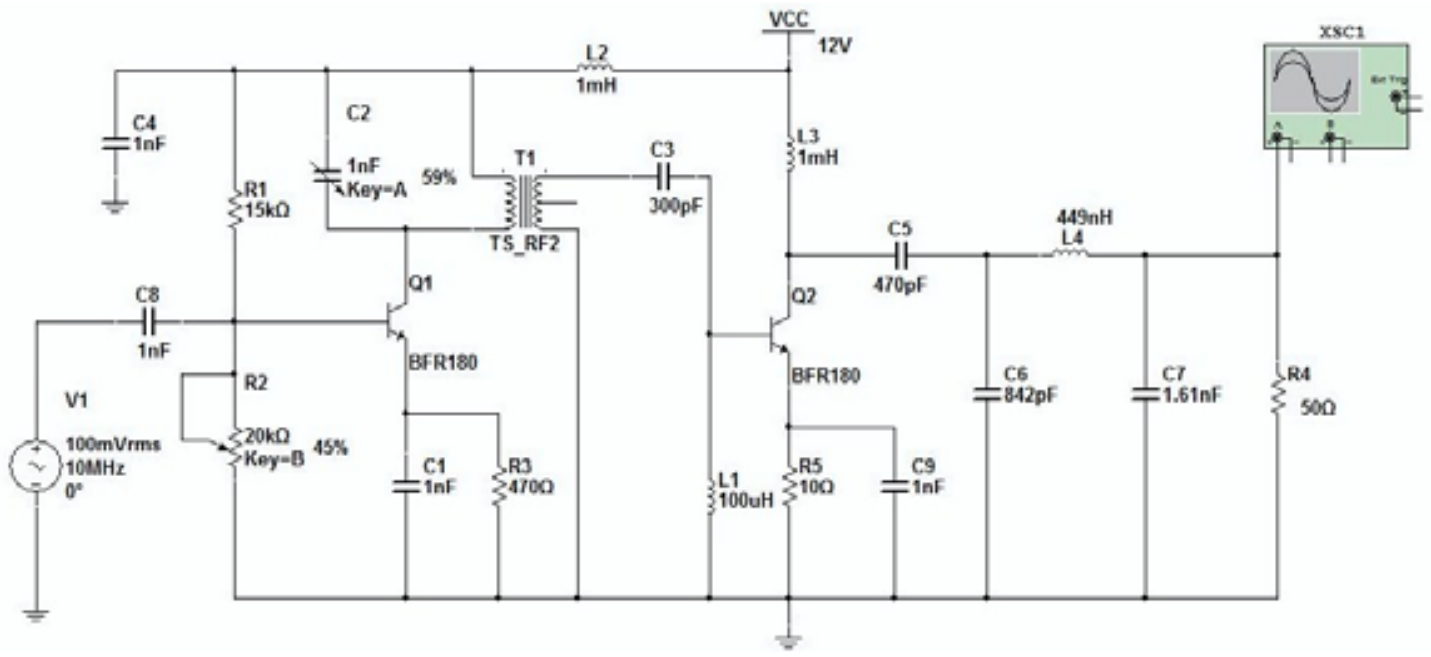

Figure 2. Principle of Class C Power Amplifier Circuit 

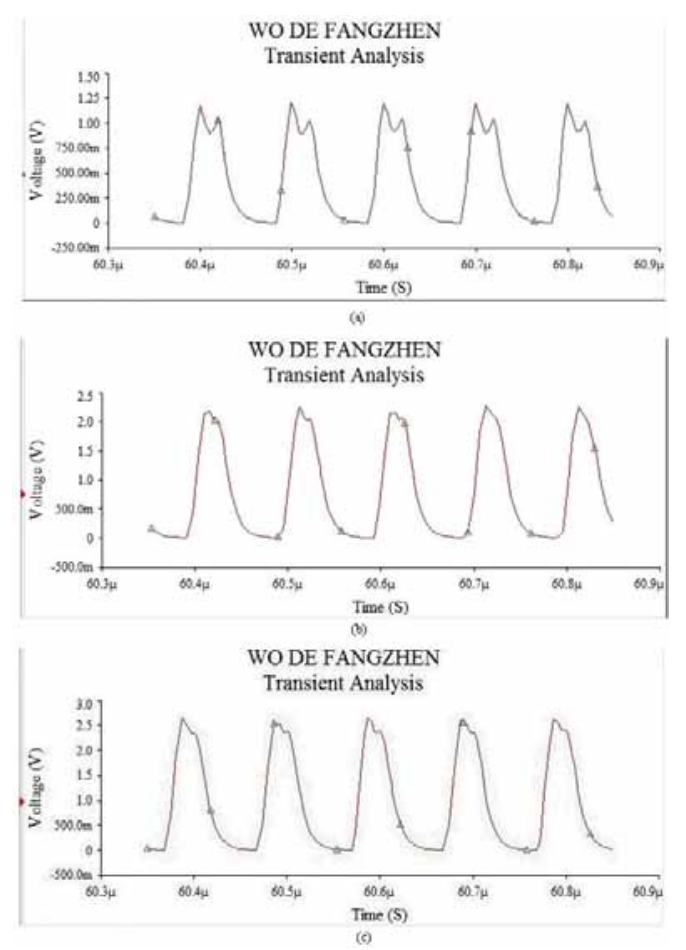

Figure 3. (a) Collector Current Oscillogram of Vcc=12V; (b) Collector Current Oscillogram of Vcc=26V; (c) Collector Current Oscillogram of $\mathrm{Vcc}=30 \mathrm{~V}$
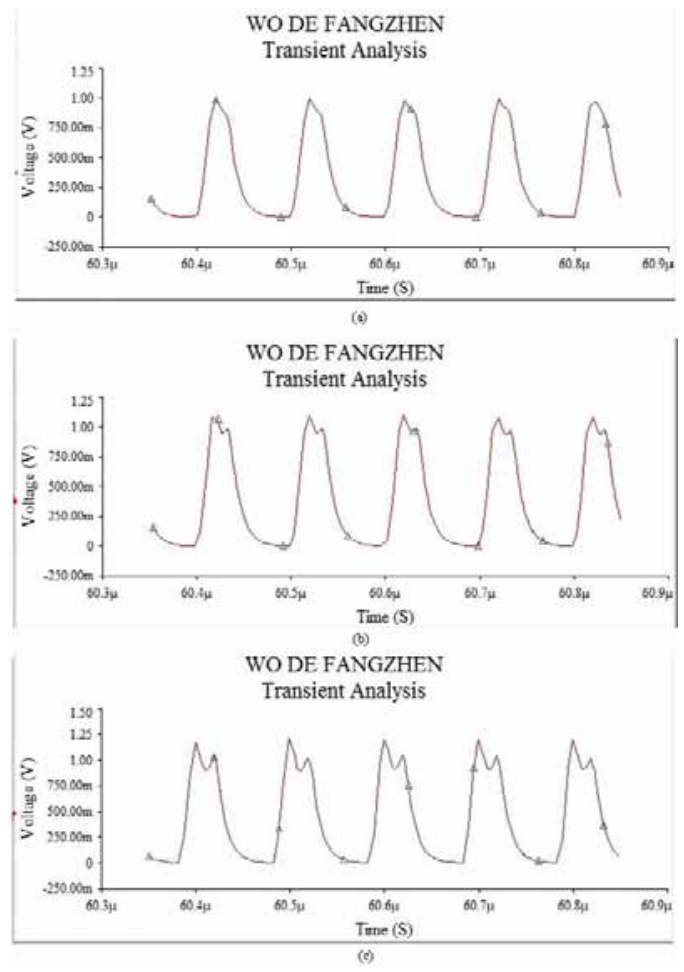

Figure 4. (a) Collector Current Oscillogram of Ubm=40mV; (b) Collector Current Oscillogram of Ubm=70mV; (c) Collector Current Oscillogram of Ubm $=100 \mathrm{mV}$ 

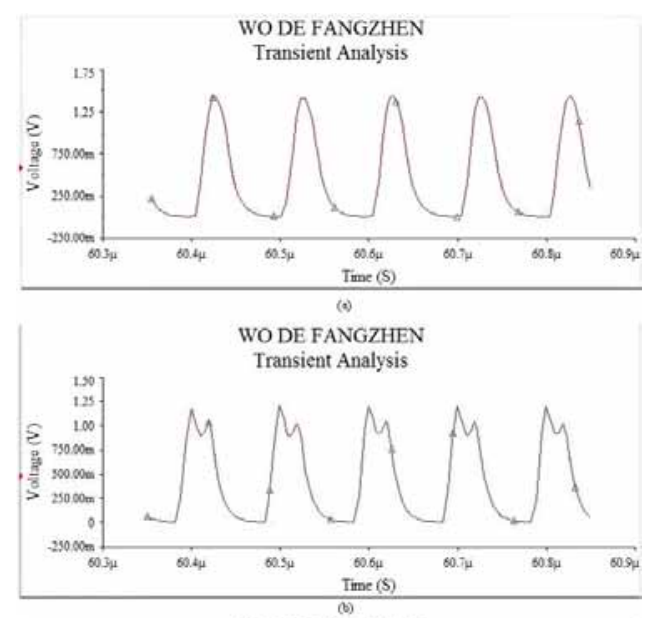

WO DE FANGZHEN

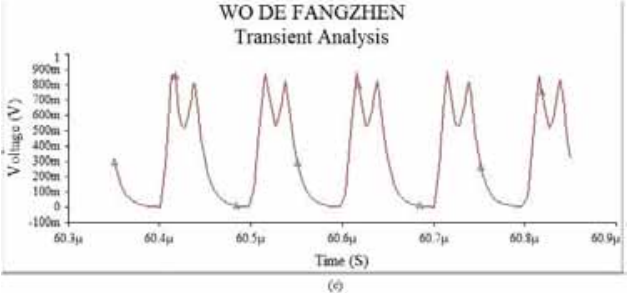

Figure 5. (a) Oscillogram of $\mathrm{R}=10 \Omega$; (b) Oscillogram of $\mathrm{R}=50 \Omega$; (c) Oscillogram of $\mathrm{R}=100 \Omega$ 